\title{
Timing of repair and mesh use in traumatic abdominal wall defects: a systematic review and meta-analysis of current literature
}

Steffi Karhof ${ }^{*}$ (D), Rianne Boot ${ }^{\dagger}$, Rogier K. J. Simmermacher, Karlijn J. P. van Wessem, Luke P. H. Leenen and Falco Hietbrink

\begin{abstract}
Background: Traumatic abdominal wall hernias or defects (TAWDs) after blunt trauma are rare and comprehensive literature on this topic is scarce. Altogether, there is no consensus about optimal methods and timing of repair, resulting in a surgeon's dilemma. The aim of this study was to analyze current literature, comparing (1) acute versus delayed repair and (2) mesh versus no mesh repair.

Methods: A broad and systematic search was conducted in PubMed, EMBASE, and the Cochrane Library. The selected articles were assessed on methodological quality using a modified version of the CONSORT 2010 Checklist and the Newcastle-Ottawa scale. Primary endpoint was hernia recurrence, diagnosed by clinical examination or CT. Random effects meta-analyses on hernia recurrence rates after acute versus delayed repair, and mesh versus no mesh repair, were conducted separately.

Results: In total, 19 studies were evaluated, of which 6 were used in our analysis. These studies reported a total of 229 patients who developed a TAWD, of whom a little more than half underwent surgical repair. Twenty-three of 172 patients (13\%) who had their TAWD surgically repaired developed a recurrence. In these studies, nearly 70\% of the patients who developed a recurrence had their TAWD repaired primarily without a mesh augmentation and mostly during the initial hospitalization. Pooled analysis did not show any statistically significant favor for either use of mesh augmentation or the timing of surgical repair.

Conclusion: Although $70 \%$ of the recurrences occurred in patients without mesh augmentation, pooled analysis did not show significant differences in either mesh versus no mesh repair, nor acute versus delayed repair for the management of traumatic abdominal wall defects. Therefore, a patient's condition (e.g., concomitant injuries) should determine the timing of repair, preferably with the use of a mesh augmentation.
\end{abstract}

Keywords: Traumatic abdominal wall defect, Traumatic defect, TAWD, Traumatic hernia

\section{Background}

Blunt traumatic abdominal wall defects (TAWDs; also known as traumatic abdominal wall hernias, TAWHs) are uncommon; its reported prevalence is less than $1 \%$ after blunt abdominal trauma [1-4]. The mechanism of injury involves a sudden and large impact-such as a seatbelt which digs into the abdomen due to a sudden deceleration after a car collision-leading to shear stress

\footnotetext{
* Correspondence: steffikarhof@gmail.com

${ }^{\dagger}$ Steffi Karhof and Rianne Boot contributed equally to this work.

Trauma Surgery Department, University Medical Centre Utrecht, PO Box 85500, 3508, GA, Utrecht, The Netherlands
}

and an elevated intra-abdominal pressure, eventually disrupting the abdominal wall [5-8].

The rarity of TAWD detection in trauma care is mostly due to the fact that less than $50 \%$ of all traumatic abdominal defects present with classical symptoms such as reducibility [9-11]. Moreover, they are often masked by superficial injuries such as hematomas or small skin defects $[12,13]$. Ultimately, serious concomitant injuries are prioritized at primary care, and there is often no time for scrutinizing the abdominal wall $[4,8,14]$. Most patients who are brought to a level I trauma center have concomitant (intra-abdominal) injuries requiring surgery.

(c) The Author(s). 2019 Open Access This article is distributed under the terms of the Creative Commons Attribution 4.0 International License (http://creativecommons.org/licenses/by/4.0/), which permits unrestricted use, distribution, and 
Although TAWDs are rare, the detection rate has increased over the past few years due to a more widely availability and more liberal use of computed tomography (CT) scans in trauma care $[1,3]$. Owing to this increase in reported incidence, the debate about the clinical significance and management of TAWDs is highly relevant. However, there is a lack of comprehensive literature on (the management of) TAWDs; it is mainly limited to case reports and a few case series and reviews [3, 13, 15]. Moreover, these publications describe a variety of management strategies, including differences in methods of repair such as mesh use $[1,3,4,13,16-20]$. Ultimately, the repair of TAWDs is not standardized. Therefore, when a TAWD is diagnosed, many surgeons are still confronted with a dilemma and the therapeutic strategy is the result of personal experience and insight $[1,5,17,21]$.

The aim of this study was to analyze current literature on the management strategies and outcomes of TAWDs. To achieve this, a thorough review and meta-analysis of current literature on this topic was carried out, focusing on acute versus delayed repair and mesh versus no mesh use.

\section{Materials and methods}

\section{Search strategy}

A broad and systematic search for all articles about TAWHs in PubMed, Embase, and the Cochrane Library was conducted by two reviewers (SK, RB) independently. The search syntax consisted of synonyms and $\mathrm{MeSH} /$ Emtree terms for traumatic abdominal wall hernias, as shown in Appendix 1 and Appendix 2. The literature search was not restricted to a certain period of time.

After removal of duplicates, all articles were screened on titles and abstracts, and irrelevant articles were excluded. After that, a full-text screening of the remaining articles was performed with predefined exclusion criteria. Studies that included outcome parameters were included; thus, studies that only reported on radiological findings were excluded, as well as studies without hernia recurrence rate as primary outcome. Moreover, articles were excluded when the full text was not available and language was limited to English, Dutch, or German. In case of disagreement of both reviewers, consensus was reached by discussion. All case series describing five patients or less were excluded as well.

\section{Methodological quality assessment}

The selected articles were assessed on methodological quality by two reviewers independently (SK, RB), using a modified version of the CONSORT 2010 Checklist developed by the Consolidated Standards of Reporting Trials (CONSORT) Group [22]. Although this checklist was originally designed for reporting randomized controlled trials, it was adapted to make it appropriate for quality assessment of the selected articles. We also completed the Newcastle-Ottawa scale, which provides some more information on specific cohort studies [23]. In case of any doubt or disagreement, consensus was reached during an expert meeting.

\section{Data extraction}

Data extraction was performed by two reviewers independently. The following data were extracted: first author, year and journal of publication, study design, country of study, number of TAWDs reported, size of surgical treatment groups, recurrence rates, and data about mortality and loss to follow-up. In particular, all articles were scrutinized for information on the timing of repair-whether this was performed during initial hospitalization or notand the use of a mesh. Data on the location of the hernia was too scarce to encounter for further analysis.

\section{Definition of timing of hernia repair}

The definitions of timing of repair differed between the included studies. Only Coleman et al. described well-defined periods of time. Hernia repair within 2 weeks post-trauma was classified as acute. The other four studies did not use these clear-cut definitions. Netto et al. and Honaker et al. used "acute" for repair during initial hospitalization, and "delayed" for all elective procedures any time afterwards. We used the definition of 2 weeks following the trauma as acute, and any time afterwards as delayed.

The corresponding authors of the included articles were contacted in case of insufficient or contradictory information.

\section{Hernia classifications}

Throughout the past years, several TAWD classification systems have been developed, based on the mechanisms of injury $[24,25]$, or considering hernia characteristics such as size or location [26, 27]. More recently, Dennis et al. [2] proposed a comprehensive grading system based on the anatomical layers of the abdominal wall. This classification is used in this article, since it allows a description of the severity of injury to the abdominal wall musculature and potential herniation of abdominal contents (Appendix 3).

\section{Outcome measure}

The main point of interest of this literature review was hernia recurrence, diagnosed either on physical examination or on computed tomography (CT) scans.

\section{Statistical analysis}

All statistical analyses were performed using Review Manager 5 (RevMan 5, Cochrane Collaboration, Copenhagen, Denmark; 2014). Meta-analyses on hernia recurrence rates after mesh versus no mesh repair, and acute versus delayed repair were conducted separately. Heterogeneity amongst the included studies was assessed using the overall effect $Z$-test and additionally tau-squared $\left(T^{2}\right)$, chi- 
squared $\left(\chi^{2}\right)$, degrees of freedom (df), and $I^{2}$ statistical measures; all were calculated using the Review Manager program. Fixed effects were used since the $I^{2}$ was equal to or less than $25 \%$ in all cases.

Because of computational difficulties in sub-analysis groups without hernia recurrences ("zero-cell counts"), the Mantel-Haenszel method was used to calculate odds ratios (OR) with $95 \%$ confidence intervals (95\% CI) [28]. $P$ values below 0.05 were considered statistically significant.

\section{Results}

\section{Search}

A search was performed at the end of December 2018 (Appendix 2). In total, 3043 articles were retrieved (Fig. 1). After removal of duplicates $(n=305)$ and exclusion on titles and abstracts $(n=2318), 420$ remaining articles were full text assessed. Almost half of them were case reports and were excluded for this reason. Moreover, no full texts could be obtained from 168 articles. Three articles (Danto; Fullerton; Gupta et al. [11]) were not included because they did not account for recurrence rates. Finally, a total of 19 articles were selected. Cross-reference checking of this selection did not yield any other reviews on the management of TAWDs. As described previously in the methods section, all case series were excluded for final analysis to reduce heterogeneity. Hereafter, 6 articles remained for meta-analysis.

\section{Baseline characteristics}

The baseline characteristics of the included studies are shown in Table 1. All selected articles had a retrospective study design and studied current practices in TAWD management and associated injuries requiring surgery. Moreover, all studies excluded patients with an abdominal wall defect after penetrating trauma, except for Park et al. [31] who included 9 patients with abdominal wall defects with 8 of them after blunt and 1 following penetrating trauma. Altogether they included a total of 273 patients with TAWDs.

\section{Methodological quality assessment}

The included studies were critically appraised with a low inter-observer variation on their methodological quality using the predefined criteria, as summarized in Additional file 1 for the adjusted CONSORT checklist and Additional file 2 for the Newcastle-Ottawa scale. Overall, only Honaker et al. [4] study was graded with a high-moderate quality of evidence; the five other articles scored between moderate-low and very low in the CONSORT checklist, and with the Newcastle-Ottawa scale, all studies scored poor quality.

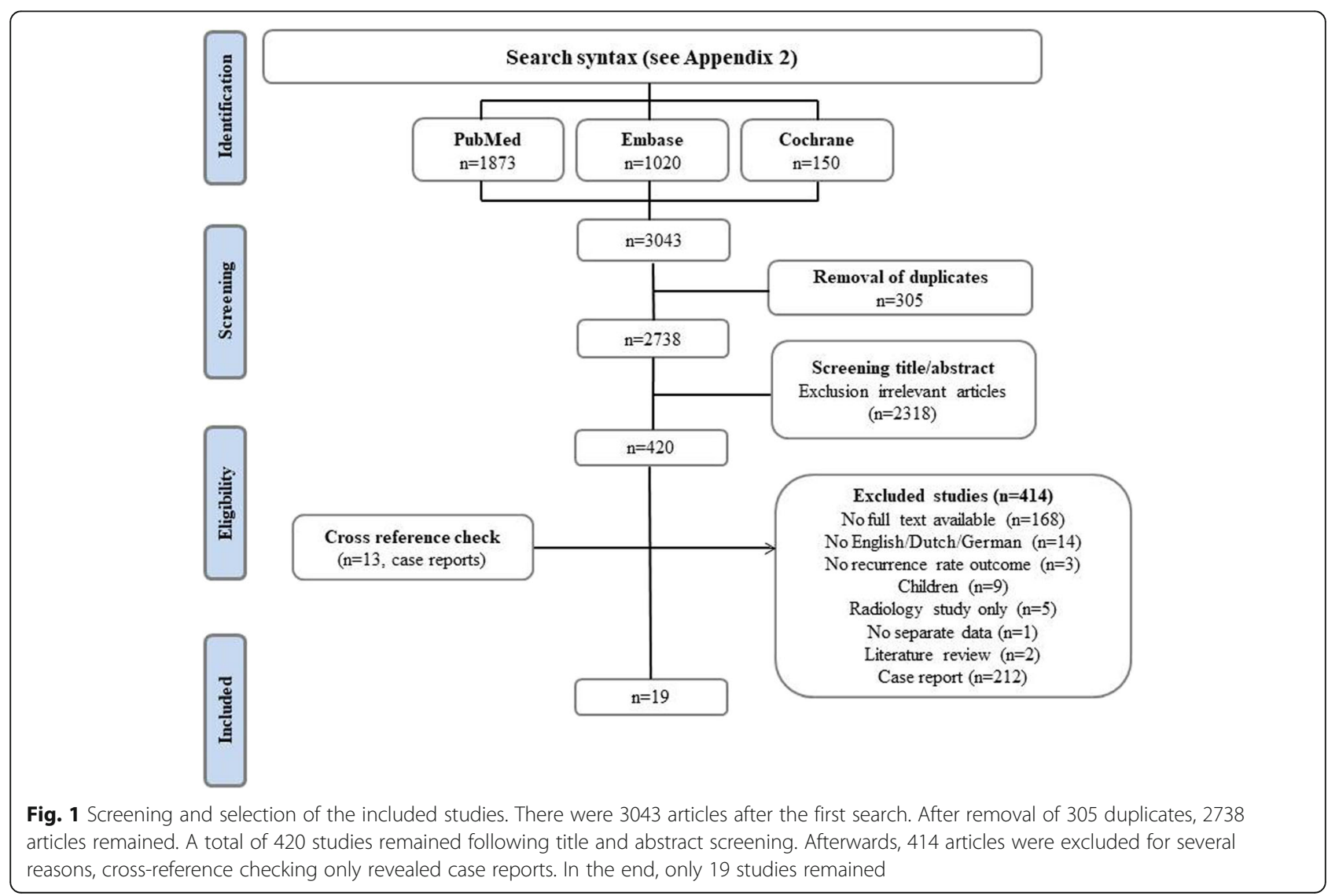


Table 1 Baseline characteristics included studies

\begin{tabular}{|c|c|c|c|c|c|c|c|c|}
\hline Author & Year & Journal & Country & $\begin{array}{l}\text { Study } \\
\text { period }\end{array}$ & $\begin{array}{l}\text { Study } \\
\text { population }\end{array}$ & $\begin{array}{l}\text { TAWDs meeting inclusion } \\
\text { criteria }\end{array}$ & TAWD type & $\begin{array}{l}\text { Surgery } \\
(\%)\end{array}$ \\
\hline Park & 2018 & Ann Surg Treat Res & Korea & $2006-2015$ & 9 & 8 & All lumbar & $8(89)$ \\
\hline Pardhan & 2016 & World J Surg & Australia & 2003-2013 & 44 & 44 & $\mathrm{~nm}$ & $41(93)$ \\
\hline Coleman & 2015 & $\begin{array}{l}\text { J Trauma Acute Care } \\
\text { Surg }\end{array}$ & USA & 2002-2014 & 80 & 80 & All types & $23(29)$ \\
\hline Honaker & 2014 & $\begin{array}{l}\text { J Trauma Acute Care } \\
\text { Surg }\end{array}$ & USA & 2007-2012 & 38 & 38 & All types & $30(79)$ \\
\hline Bender & 2008 & Am J Surg & USA & $2001-2007$ & 25 & 25 & All types & $22(88)$ \\
\hline Netto & 2006 & J Trauma & Canada & 2000-2004 & 34 & 34 & $\begin{array}{l}\text { Mainly } \\
\text { posterior }\end{array}$ & $10(29)$ \\
\hline $\begin{array}{l}\text { Vijayalakshmi } \\
\text { [29] }\end{array}$ & 2018 & J Clin Diagn Res & India & $\mathrm{nm}$ & 4 & 4 & All types & $4(100)$ \\
\hline Akbaba & 2015 & Indian J Surg & $\mathrm{nm}$ & $\mathrm{nm}$ & 3 & 3 & $\mathrm{~nm}$ & $2(33)$ \\
\hline Guttenridge & 2014 & ANZ J Surg & Australia & 2007-2010 & 5 & 5 & All types & $4(80)$ \\
\hline Singal & 2011 & J Emerg Trauma Shock & India & $\mathrm{nm}$ & 3 & 3 & All types & $3(100)$ \\
\hline Agarwal & 2009 & J Med Case Rep & India & $\mathrm{nm}$ & 2 & 2 & All types & $2(100)$ \\
\hline Kumar & 2004 & Hernia & India & $\mathrm{nm}$ & 2 & 2 & All types & $2(100)$ \\
\hline Burt & 2004 & J Trauma & USA & $\mathrm{nm}$ & 3 & 3 & Posterior & $3(100)$ \\
\hline Brenneman & 1995 & J Trauma & Canada & 1992-1993 & 9 & 9 & All types & $7(78)$ \\
\hline Damschen & 1994 & J Trauma & USA & $\mathrm{nm}$ & 5 & 4 & All types & $2(50)$ \\
\hline Fullerton & 1984 & J Emerg Med & USA & $\mathrm{nm}$ & 2 & 2 & All types & $2(100)$ \\
\hline Guly & 1983 & J Trauma & UK & $\mathrm{nm}$ & 2 & 2 & All types & $2(100)$ \\
\hline Danto & 1976 & J Trauma & USA & $\mathrm{nm}$ & 3 & 3 & All types & $3(100)$ \\
\hline Payne [30] & 1973 & J Trauma & USA & $\mathrm{nm}$ & 2 & 2 & All types & $2(100)$ \\
\hline
\end{tabular}

This table shows all the characteristics of the included studies. In most studies, the majority of patients are treated surgically for their TAWD except for Coleman, Netto, and Akbaba et al. The six studies on top of the table are included in the meta-analysis

$n m$ not mentioned

\section{Treatment of TAWD}

In four of the studies that were included for analyses, most (> 75\%) of the TAWDs were surgically repaired $[3,4,18$, 31]. Only the studies of Coleman et al. [17] and Netto et al. [1] reported low surgical repair rates of $29 \%$ both. All of the included studies reported on a diversity of reinforcement materials, including synthetic and biologic meshes. Besides these materials, Bender et al. [18] used acellular dermis of cadavers, and Brenneman et al. [19] reported on the use of autogenic tissue. Since Brenneman et al. [19] used various different treatment strategies with different muscle flaps to cover the defect, we did not include this study for further analysis on recurrence.

\section{Type of repair}

Only Honaker et al .[4]. accounted for the decision between primary and mesh repair. In this study, defects were repaired primarily when there was sufficient tissue to achieve a tension-free reconstruction. A mesh was used when a tension-free closure could not be accomplished, or when this was preferred by the surgeon over primary repair. The use of a mesh was only contraindicated in patients with abdominal contamination who had their defect repaired at initial surgery.

\section{Recurrence rates}

Overall, no significant difference between the use of mesh and no mesh was seen in all included studies as shown in Fig. 2 (pooled OR 0.55 [95\% CI 0.17-1.80]; $p=0.32$ ). Similarly, none of the studies reported significant differences with regard to the timing of repair. No favor of acute or delayed repair was demonstrated, with an overall OR of 2.47 (95\% CI 0.55-11.12; $p=0.24$ ) (Fig. 3).

Twenty-three $(13.5 \%)$ patients who underwent surgical repair showed recurrence (Table 2). The majority of them $(n=16.70 \%)$ had their TAWDs repaired without (mesh) reinforcement (Table 2). The other seven recurrences occurred in patients who had biologic meshes $(n=3)$, synthetic meshes $(n=2)$, or an absorbable mesh $(n=1)$ placed, with in one case no further description of the type of mesh.

Location of the hernia has not been mentioned in case of recurrence rates; therefore, there can be no conclusion on whether location of the hernia is relevant for recurrence. 


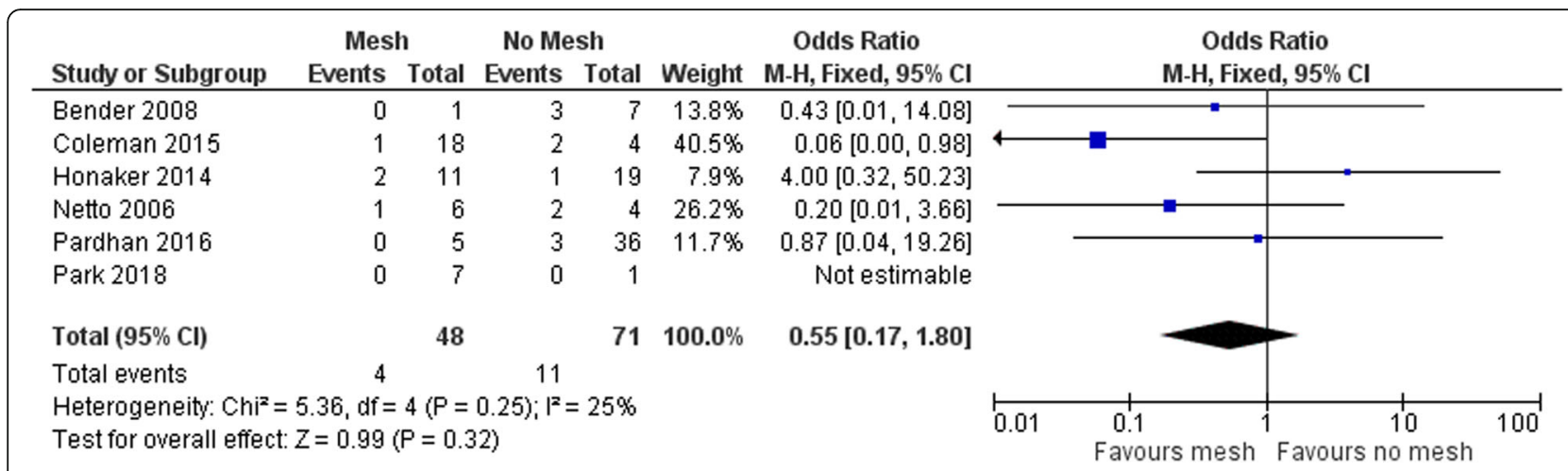

Fig. 2 Hernia recurrence (mesh vs. no mesh). The odds ratio for hernia recurrence in the six studies included in the meta-analysis, revealing no significant difference for mesh or no mesh, with a total odds ratio of 0.55 and a $95 \% \mathrm{Cl}$ of $0.17-1.80$

\section{Discussion}

This review of the literature shows that in most of the included patients who got a recurrence after surgical repair, the defect was repaired without mesh augmentation (70\%) and during the acute posttraumatic period, defined as within 2 weeks after trauma. Despite this finding, based on our pooled analysis, neither a significant favor of mesh versus no mesh use, nor a significant difference between acute and delayed repair was demonstrated.

The current available literature on this topic is scarce. It is mainly limited to case reports and a few case series and literature reviews. We only found 6 retrospective studies which investigated current practices in TAWD management, including a total of 230 patients.

Most patients had their TAWDs repaired surgically, except for the studies conducted by Netto et al. [1] and Coleman et al. [17]. In these studies, only a small part of the study population (29\%) underwent surgical repair. Based on their results, the authors advocated that operative exploration is not obligatory. They proposed conservative management in selected patients with asymptomatic defects who do not have associated injuries requiring urgent surgery $[1,17]$. This recommendation, however, may be based on a low frequency of intra-abdominal injuries reported by
Coleman et al. [17]. Moreover, 2 of the 26 patients who were initially managed non-operatively by Netto et al. [1] developed symptomatic defects and underwent secondary surgical repair. Ultimately, both studies did not classify the TAWDs based on the classification system provided by Dennis et al. [2]. Therefore, the severity of the injury to the abdominal wall musculature and-most important-potential herniation of abdominal contents could not be assessed.

When evaluating the results in the patient population that underwent surgery, two measures were studied: mesh use and timing of repair. Honaker et al. [4] noticed no recurrences at all in the patient group which underwent mesh repair. Nevertheless, four of the studies in our literature review did not report a significant favor for mesh or no mesh use, mainly due to a lack of power owing to small study sample sizes [1, 3, 4, 17]. Only Bender et al. [18] mentioned a significant difference; however, they used different reinforcements including absorbable meshes and the acellular dermis of cadavers, resulting in a heterogeneous comparison.

After all, repairing techniques according to current general principles (tension-free repair) are recommended to minimize recurrence rates $[3,18]$. If this cannot be achieved primarily-this may be especially difficult in

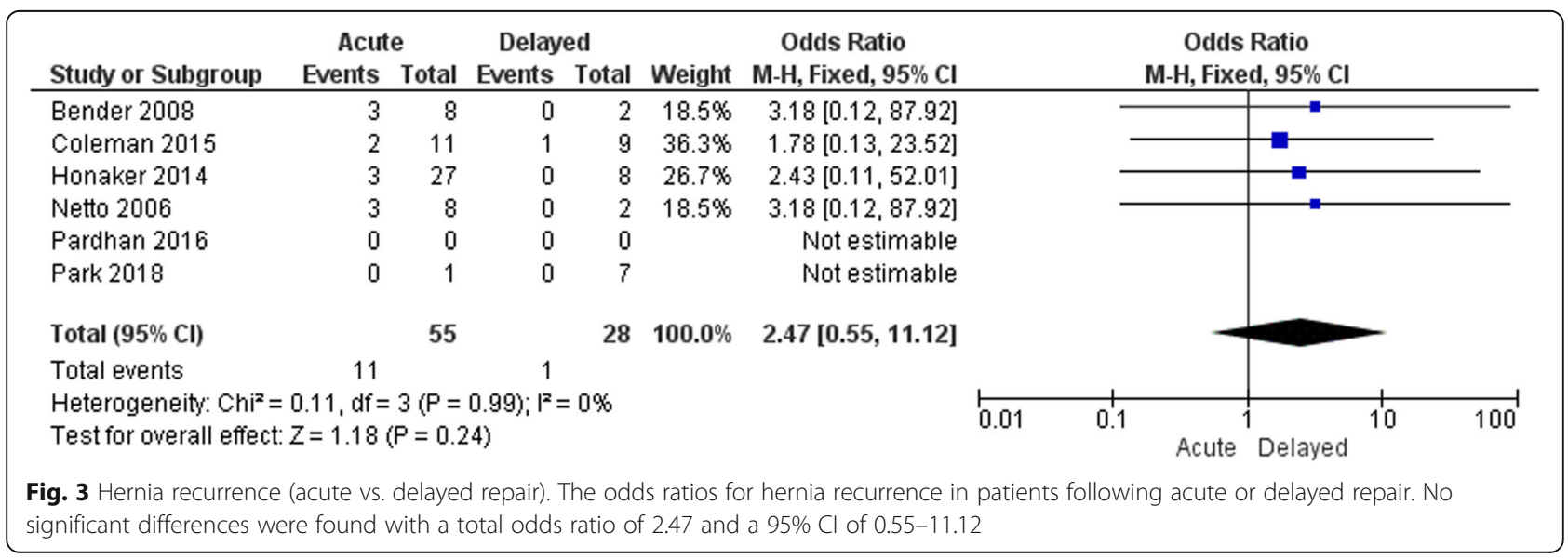


Table 2 Outcome

\begin{tabular}{|c|c|c|c|c|c|c|c|c|c|}
\hline \multicolumn{3}{|l|}{ Characteristics } & \multicolumn{4}{|c|}{ Surgical repair } & \multicolumn{3}{|l|}{ Outcome } \\
\hline Author & Median ISS (range) & TAWDs & Total (\%) & Acute & Delayed & Mesh & Recurrence rate (mesh used) & Mortality (\%) & Loss to FU (\%) \\
\hline Park & $\mathrm{nm}$ & 9 & $8(89)$ & 0 & 7 & 7 & 0 & 0 & 0 \\
\hline Pardhan & $23(\mathrm{~nm})$ & 44 & $41(93)$ & 8 & 33 & 5 & $3(0)$ & $4(9)$ & $1(2)$ \\
\hline Coleman & $22(\mathrm{~nm})$ & 80 & $23(29)$ & 18 & 5 & 7 & $6(3)$ & 0 & $\mathrm{~nm}$ \\
\hline Honaker & $17(1-66)$ & 38 & $30(79)$ & 27 & 3 & 11 & $3(2)$ & $2(5)$ & 0 \\
\hline Bender & $35(\mathrm{~nm})$ & 25 & $22(88)$ & 11 & 11 & $18^{*}$ & $3(1)$ & $1(4)$ & $3(12)$ \\
\hline Netto & $31(18-44)$ & 34 & $10(29)$ & 8 & $2^{* *}$ & 1 & $3(0)$ & $1(3)$ & $11(32)$ \\
\hline Vijayalakshmi & $\mathrm{nm}$ & 4 & $4(100)$ & 4 & 0 & 0 & 0 & 0 & 0 \\
\hline Akbaba & $\mathrm{nm}$ & 3 & $2(33)$ & 0 & 2 & 2 & 0 & 0 & 0 \\
\hline Guttenridge & $14(9-29)$ & 5 & $4(80)$ & 3 & 1 & 2 & 0 & 0 & $1(20)$ \\
\hline Singal & $\mathrm{nm}$ & 3 & $3(100)$ & 3 & 0 & 1 & 0 & 0 & 0 \\
\hline Agarwal & $\mathrm{nm}$ & 2 & $2(100)$ & 2 & 0 & 1 & 0 & 0 & 0 \\
\hline Kumar & $\mathrm{nm}$ & 2 & $2(100)$ & 2 & 0 & 0 & 0 & 0 & 0 \\
\hline Burt & $\mathrm{nm}$ & 3 & $3(100)$ & 0 & 3 & 3 & $1(1)$ & 0 & $\mathrm{~nm}$ \\
\hline Brenneman & nm (mean 25) & 9 & $7(78)$ & 2 & 5 & 5 & $2(0)$ & $\mathrm{nm}$ & $\mathrm{nm}$ \\
\hline Damschen & $\mathrm{nm}$ & 4 & $2(50)$ & 2 & 0 & 0 & $1(0)$ & $\mathrm{nm}$ & $2(50)$ \\
\hline Fullerton & $\mathrm{nm}$ & 2 & $2(100)$ & 2 & 0 & $\mathrm{~nm}$ & 0 & $1(50)$ & $\mathrm{nm}$ \\
\hline Guly & $\mathrm{nm}$ & 2 & $2(100)$ & 1 & 1 & 0 & 0 & 0 & 0 \\
\hline Danto & $\mathrm{nm}$ & 3 & $3(100)$ & 3 & 0 & $\mathrm{~nm}$ & $\mathrm{~nm}$ & 1 (33) & $\mathrm{nm}$ \\
\hline Payne & $\mathrm{nm}$ & 2 & $2(100)$ & 1 & 1 & 0 & $1(0)$ & 0 & $\mathrm{~nm}$ \\
\hline Total & NA & 274 & $172(63)$ & 98 & 74 & 63 & $23(7)$ & $10(\mathrm{NA})$ & $18(\mathrm{NA})$ \\
\hline
\end{tabular}

This table shows surgical repair and outcome for all 19 studies. From a total of 274 patients with TAWDs, 172 underwent surgical repair, both acute ( $n=98)$ and delayed $(n=74)$, with a minority of mesh repair $(n=63)$. A total of 23 recurrences occurred in this patient group $n m$ not mentioned, NA not applicable

*Reinforcement consists of absorbable mesh, permanent mesh, and acellular cadaver dermis

**Two of the patients who were initially treated conservatively developed symptoms requiring surgery after 8 months

the acute posttraumatic period due to swelling and hematoma-a mesh should be used.

In addition to this, both Liasis et al. [16] and Bender et al. recommended mesh use in all patients with delayed TAWD repair, although they questioned mesh repair in emergency settings due to potential contamination. Contamination is considered to be a relative contra-indication for non-absorbable mesh use, due to an increased risk of infectious complications, eventually potentially mesh removal [3, 32-34].

Coleman et al. mentioned the use of open abdomen treatment for contamination in six patients following their primary surgery. Honaker et al. also mentioned an average number of 2.1 operations before TAWD repair in patients requiring damage control surgery at first.

In addition to contamination as a possible cause for delayed surgery, the physiological condition of the patient could also play a role in the decision to possibly postpone repair of the abdominal wall defect. Unfortunately, the information in most of the studies is limited, so these variables could not be included in our analysis.

Concerning the timing of repair, there was no significant difference between repair at initial trauma laparotomy or in an elective setting during hospitalization. By contrast, Honaker et al. [4] reported that all recurrences occurred in the group that underwent immediate repair. Moreover, Brenneman et al. [19], who repaired seven TAWDs at initial trauma laparotomy, observed that acute repair without mesh failed in a majority of the patients (5/7). Based on these results, it is not possible to make a strong recommendation for the optimal timing of repair though, since these studies were very heterogeneous in their description of timing of repair. Besides that, none of them demonstrated a significant difference between the acute and delayed repair groups. Ultimately, the choice for the timing of repair is mainly dependent on (the extent of) concomitant findings [16]. Early repair may be dictated by the extent of the injury and concomitant injuries, and therefore, the choice for management strategy must be based on individual circumstances [3, 17]. Moreover, as one may suggest, an early TAWD repair decreases the risk of bowel obstruction, strangulation, and/or incarceration $[1,5,7,9,35]$.

To our knowledge, this is the second largest review on the treatment strategies of TAWDs. Since TAWDs are rare, there are only limited amounts of case reports, series, and reviews evaluating different treatment strategies. The first 
literature review was published in 2003 by Liasis et al. and included 145 articles with a total of 248 TAWD cases. However, this review included mainly case reports and case reviews, leading to a more heterogeneous study population.

Liasis et al. do propose a very helpful treatment algorithm in which all TAWDs are surgically repaired. Furthermore, they noted that timing and mesh repair is situation-dependent. They recommended mesh use in all patients with delayed TAWD repair, but they questioned mesh repair in emergency settings due to potential contamination. Concerning the timing of repair, in cases where neither an emergency laparotomy is required nor a risk of incarceration is present, Liasis et al. state that delayed repair is justified.

In comparison with Liasis et al., our literature review provides insight into a thorough search in current literature, excluding case reports and case series. This resulted in a more homogenous treated patient population. In our comprehensive review, it is noted that most of the recurrences occur in the patients following primary repair instead of with a mesh, even though this does not show in the pooled analysis.

Timing does not seem to be an important predictor for recurrence, making a patient's physiology (condition and concomitant injuries) the most important factor in determining the timing of repair.

There are several limitations to this study. First of all, these results are still based on relatively small numbers of patients. Therefore, the recommendations regarding optimal timing of repair and mesh use should be interpreted carefully. The variation in follow-up duration (1-60 months) across the selected studies also affected the outcome of the pooled results. Secondly, regarding the methodological quality of the included studies, only Honaker et al. [4] was graded with a high-moderate quality of evidence; the five other articles scored between moderate-low and very low. Besides that, the selected articles did neither account extensively for concomitant injuries nor the size of the defect and presence of herniation. Moreover, differences in definitions of timing of repair and a variety of mesh types led to a heterogeneous study population, possibly leading to selection bias. In addition to this, the risk of bias has been increased by Netto et al. who reported a striking loss to follow-up (32\%). Lastly, a lack of information on the choice for the treatment strategy (primary versus mesh repair; acute versus delayed repair) made it impossible to propose a treatment algorithm.

\section{Conclusion}

Although not statistically significant in a pooled analysis, it is noted that $70 \%$ of the recurrences were found in patients without the use of mesh augmentation during repair. Furthermore, it is important to note that timing is not an important risk factor for recurrence, which makes the choice for the timing of repair highly dependent on the individual circumstances of each patient. Most patients have multiple concomitant injuries, but no increased risk was found for early repair after trauma. A larger, multicenter prospective cohort study is required to evaluate recurrence rates after TAWD repair.

\section{Appendix 1}

\section{Search terms}

TAWH

TAWHs

Handlebar hernia

Handlebar hernias

Handlebar herniation

Handlebar herniations

Handlebar injury

Handlebar injuries

Handlebar trauma

Trauma

Wounds and injuries [MESH terms]

Traumatic

Posttraumatic

Seat belt

Seat belts [MESH terms]

Seatbelt

Seatbelts

Seat-belt

Seat-belts

Abdominal wall

Abdominal wall [MESH terms]

Abdominal-wall

Abdominal fascia

Abdominal muscle

Abdominal muscles

Abdominal muscles [MESH terms]

Abdominal musculature

Abdominal wall musculature

Hernia

Hernias

Hernia, abdominal [MESH terms]

Hernia, ventral [MESH terms]

Hernia, inguinal [MESH terms]

Herniation

Herniations

Avulsion

Avulsions

Defect

Defects

Disruption

Disruptions

Injury

Injuries

Rupture

Ruptures

Hernia [MESH terms] 


\section{Appendix 2}

Table 3 Search syntax

\begin{tabular}{lll}
\hline Database & Syntax & Hits \\
\hline Pubmed & \#1 OR (\#2 AND \#3 AND \#4) & 1873
\end{tabular}

\#1: ((()(((TAWH[Title/Abstract]) OR TAWHs[Title/Abstract]) OR “Handlebar hernia"[Title/Abstract]) OR "Handlebar hernias"[Title/

(31-12-2018) Abstract]) OR "Handlebar herniation"Title/Abstract]) OR "Handlebar herniations" [Title/Abstract]) OR "Handlebar injury"[Title/

Abstract]) OR "Handlebar injuries"[Title/Abstract]) OR "Handlebar trauma"[Title/Abstract]

138 hits

\#2: ((((c(((Trauma[Title/Abstract]) OR (wounds and injuries[MeSH Terms])) OR Traumatic[Title/Abstract]) OR Posttraumatic[Title/

Abstract]) OR "Seat belt"[Title/Abstract]) OR seat belts[MeSH Terms]) OR Seatbelt[Title/Abstract]) OR Seatbelts[Title/Abstract]) OR

"Seat-belt"[Title/Abstract]) OR "Seat-belts"[Title/Abstract]

\subsection{0 hits}

\#3: (((((((("Abdominal wall"[Title/Abstract]) OR abdominal wall[MeSH Terms]) OR "Abdominal-wall"[Title/Abstract]) OR "Abdominal fascia"[Title/Abstract]) OR "Abdominal muscle"[Title/Abstract]) OR "Abdominal muscles"[Title/Abstract]) OR abdominal muscles[MeSH Terms]) OR "Abdominal musculature"[Title/Abstract]) OR "Abdominal wall musculature"[Title/Abstract]

\subsection{7 hits}

\#4: $(((c(c(c(c(c((($ Hernia[Title/Abstract])) OR Hernias[Title/Abstract]) OR hernia, abdominal[MeSH Terms]) OR hernia, ventral[MeSH Terms]) OR hernia, inguinal[MeSH Terms]) OR Herniation[Title/Abstract]) OR Herniations[Title/Abstract]) OR Avulsion[Title/ Abstract]) OR Avulsions[Title/Abstract]) OR Defect[Title/Abstract]) OR Defects[Title/Abstract]) OR Disruption[Title/Abstract]) OR Disruptions[Title/Abstract]) OR Injury[Title/Abstract]) OR Injuries[Title/Abstract]) OR Rupture[Title/Abstract]) OR Ruptures[Title/ Abstract]) OR Hernia[MeSH Terms]

\subsubsection{2 hits}

Embase (\#1 OR (\#2 AND \#3 AND \#4)) AND [embase]/lim

(31-12-2018) \#1: 'traumatic abdominal wall hernia':ab,ti OR 'traumatic abdominal wall hernias':ab,ti OR tawh:ab,ti OR tawhs:ab,ti OR 'handlebar hernia':ab,ti OR 'handlebar hernias':ab,ti OR 'handlebar herniation':ab,ti OR 'handlebar herniations':ab,ti

165 hits

\#2: trauma:ab,ti OR traumatic:ab,ti OR posttraumatic:ab,ti OR handlebar:ab,ti OR 'seat belt':ab,ti OR 'seat belts'ab,ti OR seatbelt:ab, ti OR seatbelts:ab,ti OR 'seat-belt':ab,ti OR 'seat-belts':ab,ti

\subsection{0 hits}

\#3: 'abdominal wall':ab,ti OR 'abdominal wall'/exp OR 'abdominal-wall':ab,ti OR 'abdominal fascia':ab,ti OR 'abdominal muscle':ab,ti OR ‘abdominal muscles':ab,ti OR ‘abdominal musculature':ab,ti OR ‘abdominal wall musculature'/exp

\subsection{2 hits}

\#4: hernia:ab,ti OR hernias:ab,ti OR 'abdominal wall hernia'/exp OR herniation:ab,ti OR herniations:ab,ti OR defect:ab,ti OR defects: ab,ti OR disruption:ab,ti OR disruptions:ab,ti OR injury:ab,ti OR injuries:ab,ti OR rupture:ab,ti OR ruptures:ab,ti

1.666.090 hits

Cochrane \#1 OR (\#2 AND \#3 AND \#4)

(31-12-2018 \#1: tawh:ti,ab OR tawhs:ti,ab OR "handlebar hernia":ti,ab OR "handlebar hernias":ti,ab OR "handlebar herniation":ti,ab OR "handlebar herniations":ti,ab OR "handlebar injury":ti,ab OR "handlebar injuries":ti,ab OR "handlebar trauma":ti,ab

\section{0 hits}

\#2: trauma:ti,ab or traumatic:ti,ab or posttraumatic:ti,ab or handlebar:ti,ab or "seat belt":ti,ab or "seat belts":ti,ab or seatbelt:ti,ab or seatbelts:ti,ab or seat-belt:ti,ab or seat-belts:ti,ab

\subsection{9 hits}

\#3: "abdominal wall":ti,ab or abdominal-wall:ti,ab or "abdominal fascia":ti,ab or "abdominal muscle":ti,ab or "abdominal muscles":ti, ab or "abdominal musculature":ti,ab or "abdominal wall musculature":ti,ab or "anterior abdominal":ti,ab or flank:ti,ab or lumbar:ti, ab or inguinal:ti,ab or groin:ti,ab or spigeli:ti,ab or spigelian:ti,ab 15.442 hits

\#4: hernia:ti,ab or hernias:ti,ab or herniation:ti,ab or herniations:ti,ab or defect:ti,ab or defects:ti,ab or disruption:ti,ab or disruptions:ti,ab or injury:ti,ab or injuries:ti,ab or rupture:ti,ab or ruptures:ti,ab

45.835 hits

\section{Appendix 3}

Table 4 TAWD classification system (Dennis et al. [2])

\begin{tabular}{ll}
\hline TAWH classification system & \\
\hline Grade I & Subcutaneous tissue contusion \\
\hline Grade II & Abdominal wall muscle hematoma \\
Grade III & Single abdominal muscle disruption \\
Grade IV & $\begin{array}{l}\text { Complete abdominal wall } \\
\text { muscle disruption }\end{array}$ \\
Grade V & $\begin{array}{l}\text { Complete abdominal wall muscle disruption } \\
\text { with herniation of abdominal contents }\end{array}$ \\
Grade VI & Open herniation (evisceration) \\
\hline
\end{tabular}




\section{Supplementary information}

Supplementary information accompanies this paper at https://doi.org/10. 1186/s13017-019-0271-0

Additional file 1. Methodological quality assessment-CONSORT checklist.

Additional file 2. Predefined criteria on methodological quality assessment.

\section{Abbreviations}

Cl: Confidence interval; CT: Computed tomography; OR: Odds ratio; TAWD: Traumatic abdominal wall defect

\section{Acknowledgements}

Not applicable.

\section{Authors' contributions}

SK and RB share co first authorship to this manuscript. SK contributed to the data acquisition, analysis and interpretation, and writing and revision of the manuscript. RB contributed to the data collection, data analysis and interpretation, and writing and revision of the manuscript. RS contributed to the study concept, data interpretation, and revision of the manuscript. KW contributed to the interpretation and revision of the manuscript. LL contributed to the interpretation and writing/revision of the manuscript. FH contributed to the study design, data analysis and interpretation, and revision of the manuscript. All authors read and approved the final manuscript.

\section{Funding}

No funding has been provided to support this manuscript

\section{Availability of data and materials}

Data sharing not applicable to this article as no datasets were generated or analyzed during the current study.

\section{Ethics approval and consent to participate}

Not applicable.

\section{Consent for publication}

Not applicable.

\section{Competing interests}

The authors declare that they have no competing interests.

Received: 6 August 2019 Accepted: 9 October 2019

Published online: 17 December 2019

\section{References}

1. Netto FA, Hamilton P, Rizoli SB, et al. Traumatic abdominal wall hernia: epidemiology and clinical implications. J Trauma. 2006;61(5):1058-61.

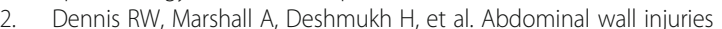
occurring after blunt trauma: incidence and grading system. Am J Surg. 2009:197(3):413-7.

3. Pardhan A, Mazahir S, Rao S, Weber D. Blunt traumatic abdominal wall hernias: a surgeon's dilemma. World J Surg. 2016;40(1):231-5.

4. Honaker D, Green J. Blunt traumatic abdominal wall hernias: associated injuries and optimal timing and method of repair. J Trauma Acute Care Surg. 2014;77(5):701-4

5. Gutteridge I, Towsey K, Pollard C. Traumatic abdominal wall herniation: case series review and discussion. ANZ J Surg. 2014;84(3):160-5.

6. Suhardja TS, Atalla MA, Rozen WM. Complete abdominal wall disruption with herniation following blunt injury: case report and review of the literature. Int Surg. 2015;100(3):531-9.

7. Yucel N, Ugras MY, Isik B, Turtay G. Case report of a traumatic abdominal wall hernia resulting from falling onto a flat surface. Ulus Travma Acil Cerrahi Derg. 2010;16(6):571-4

8. Singal R, Gupta R, Mittal A, et al. Delayed presentation of the traumatic abdominal wall hernia; dilemma in the management - review of literature. Indian J Surg. 2012;74(2):149-56.
9. Kumar A, Hazrah P, Bal S, Seth A, Parshad R. Traumatic abdominal wall hernia: a reappraisal. Hernia. 2004;8(3):277-80.

10. Lukan JK, Franklin GA, Spain DA, Richardson JD. Traumatic lumbar hernias: difficulty with diagnosis and repair. Eur J Trauma. 2000;26(2):81-4.

11. Gupta S, Dalal U, Sharma R, Dalal A, Attri AK. Traumatic abdominal wall hernia. Ulus Travma Acil Cerrahi Derg. 2011;17(6):493-6.

12. Wilson $\mathrm{KL}$, Davis MK, Rosser JC Jr. A traumatic abdominal wall hernia repair: a laparoscopic approach. JSLS. 2012;16(2):287-91.

13. Burt BM, Afifi HY, Wantz GE, Barie PS. Traumatic lumbar hernia: report of cases and comprehensive review of the literature. J Trauma. 2004; 57(6):1361-70.

14. Belgers HJ, Hulsewe KW, Heeren PA, Hoofwijk AG. Traumatic abdominal wall hernia: delayed presentation in two cases and a review of the literature. Hernia. 2005;9(4):388-91.

15. Losanoff JE, Richman BW, Jones JW. Handlebar hernia: ultrasonographyaided diagnosis. Hernia. 2002;6(1):36-8.

16. Liasis L, Tierris I, Lazarioti F, Clark CC, Papaconstantinou HT. Traumatic abdominal wall hernia: is the treatment strategy a real problem? J Trauma Acute Care Surg. 2013;74(4):1156-62.

17. Coleman JJ, Fitz EK, Zarzaur BL, et al. Traumatic abdominal wall hernias: location matters. J Trauma Acute Care Surg. 2016;80(3):390-6.

18. Bender JS, Dennis RW, Albrecht RM. Traumatic flank hernias: acute and chronic management. Am J Surg. 2008;195(3):414-7.

19. Brenneman FD, Boulanger BR, Antonyshyn O. Surgical management of abdominal wall disruption after blunt trauma. J Trauma. 1995;39(3):539-44.

20. Cornelissen MP, van Buijtenen J, van den Heuvel B, Bloemers F, Geeraedts L Jr. Blunt abdominal wall disruption by seatbelt injury; a case report and review of the literature. Bull Emerg Trauma. 2016;4(2):105-9.

21. Hamidian Jahromi A, Skweres J, Sangster G, Johnson L, Samra N. What we know about management of traumatic abdominal wall hernia: review of the literature and case report. Int Surg. 2015:100(2):233-9.

22. Moher D, Hopewell S, Schulz KF, et al. CONSORT 2010 explanation and elaboration: updated guidelines for reporting parallel group randomised trials. J Clin Epidemiol. 2010;63(8):1-37.

23. Wells G, Shea S, O'Connell D, et al. The Newcastle-Ottawa Scale (NOS) for assessing the quality of nonrandomised studies in meta-analyse. Available from http://www.ohri.ca/programs/clinical_epidemiology/oxford.asp. Accessed 22 Sept 2019.

24. Lane CT, Cohen AJ, Cinat ME. Management of traumatic abdominal wall hernia. Am Surg. 2003;69(1):73-6.

25. Moreno-Egea A, Girela E, Parlorio E, Aguayo-Albasini JL. Controversies in the current management of traumatic abdominal wall hernias. Cir Esp. 2007 82(5):260-7

26. Wood RJ, Ney AL, Bubrick MP. Traumatic abdominal hernia: a case report and review of the literature. Am Surg. 1988;54(11):648-51.

27. Clain A. Traumatic hernia. Br J Surg. 1964:51:549-50.

28. Bradburn M, Deeks J, Berlin J, Russell L. Much ado about nothing: a comparison of the performance of meta-analytical methods with rare events<br />. Stat Med. 2007 Jan;26(1):53-77.

29. Vijayalakshmi G, Kumar LA. Traumatic abdominal wall hernias - an uncommon case series. J Clin Diagn Res. 2018;12(9):PR04-5.

30. Payne DD, Resnicoff SA, States J. Williams J. J Trauma. 1973:13(3):262-7.

31. Park Y, Chung M, Lee MA. Traumatic lumbar hernia: clinical features and management. Ann Surg Treat Res. 2018;95(6):340-4.

32. Atema JJ, de Vries FEE, Boermeester MA. Systematic review and metaanalysis of the repair of potentially contaminated and contaminated abdominal wall defects. Am J Surg. 2016;212(5):982-95.

33. Choi JJ, Palaniappa NC, Dallas KB, Rudich TB, Colon MJ, Divino CM. Use of mesh during ventral hernia repair in clean-contaminated and contaminated cases: outcomes of 33,832 cases. Ann Surg. 2012;255(1):176-80.

34. Carbonell AM, Criss CN, Cobb WS, Novitsky YW, Rosen MJ. Outcomes of synthetic mesh in contaminated ventral hernia repairs. J Am Coll Surg. 2013, 217(6):991-8.

35. Mahajna A, Ofer A, Krausz MM. Traumatic abdominal hernia associated with large bowel strangulation: case report and review of the literature. Hernia. 2004:8(1):80-2.

\section{Publisher's Note}

Springer Nature remains neutral with regard to jurisdictional claims in published maps and institutional affiliations. 